\title{
Enhancing the Cognitive Effects of Flavonoids With Physical Activity: Is There a Case for the Gut Microbiome?
}

\author{
Carol L. Cheatham ${ }^{1}$, David C. Nieman' ${ }^{2}$, Andrew P. Neilson ${ }^{3}$ and Mary Ann Lila ${ }^{3 *}$ \\ 1 Department of Psychology and Neuroscience, University of North Carolina at Chapel Hill, Chapel Hill, NC, United States, \\ ${ }^{2}$ Human Performance Lab, Department of Biology, Appalachian State University, Kannapolis, NC, United States, \\ ${ }^{3}$ Department of Food, Bioprocessing and Nutrition Sciences, Plants for Human Health Institute, North Carolina State \\ University, Kannapolis, NC, United States
}

OPEN ACCESS

Edited by:

David Vauzour,

University of East Anglia,

United Kingdom

Reviewed by:

Silvia Turroni,

University of Bologna, Italy

Naiman A. Khan,

University of Illinois

at Urbana-Champaign, United States

*Correspondence:

Mary Ann Lila

mlila@ncsu.edu

Specialty section:

This article was submitted to

Gut-Brain Axis,

a section of the journal

Frontiers in Neuroscience

Received: 10 December 2021

Accepted: 20 January 2022

Published: 22 February 2022

Citation:

Cheatham CL, Nieman DC, Neilson AP and Lila MA (2022)

Enhancing the Cognitive Effects of Flavonoids With Physical Activity: Is

There a Case for the Gut

Microbiome?

Front. Neurosci. 16:833202. doi: 10.3389/fnins.2022.833202
Age-related cognitive changes can be the first indication of the progression to dementias, such as Alzheimer's disease. These changes may be driven by a complex interaction of factors including diet, activity levels, genetics, and environment. Here we review the evidence supporting relationships between flavonoids, physical activity, and brain function. Recent in vivo experiments and human clinical trials have shown that flavonoid-rich foods can inhibit neuroinflammation and enhance cognitive performance. Improved cognition has also been correlated with a physically active lifestyle, and with the functionality and diversity of the gut microbiome. The great majority $(+90 \%)$ of dietary flavonoids are biotransformed into phytoactive phenolic metabolites at the gut microbiome level prior to absorption, and these prebiotic flavonoids modulate microbiota profiles and diversity. Health-relevant outcomes from flavonoid ingestion may only be realized in the presence of a robust microbiome. Moderate-to-vigorous physical activity (MVPA) accelerates the catabolism and uptake of these gut-derived antiinflammatory and immunomodulatory metabolites into circulation. The gut microbiome exerts a profound influence on cognitive function; moderate exercise and flavonoid intake influence cognitive benefits; and exercise and flavonoid intake influence the microbiome. We conclude that there is a potential for combined impacts of flavonoid intake and physical exertion on cognitive function, as modulated by the gut microbiome, and that the combination of a flavonoid-rich diet and routine aerobic exercise may potentiate cognitive benefits and reduce cognitive decline in an aging population, via mechanisms mediated by the gut microbiome. Mechanistic animal studies and human clinical interventions are needed to further explore this hypothesis.

Keywords: age-related, cognition, neuroprotective, phenolic metabolites, prebiotic flavonoids, exercise, microbiome

\section{INTRODUCTION}

The brain can be a neglected aspect of human health. Typically, brain health is not a consideration until people reach their 50s or 60s, when brain function can become less reliable. Moreover, lifestyle choices are hardly ever considered as detrimental to brain health in an otherwise healthy adult. Importantly, the number of Americans aged 65 and older is projected to nearly double from 52 
million in 2018 to 95 million by 2060 (United States Census Bureau, 2021). As the life expectancy of the world's population increases, dementia (age-related decline from previously attained cognitive levels) is a looming threat to the individual's healthspan, and causes a heavy economic burden to families and to society. Researchers have put considerable effort into developing interventions for those who have been diagnosed with a dementia (e.g., Alzheimer's disease, Parkinson's disease, and Dementia with Lewy Bodies). We posit that validating proactive strategies to prevent cognitive dysfunction in the aging demographic should be a high priority.

In this review, we will explore the case for flavonoids as a prophylactic against declining brain health, investigate the modulating role(s) of the gut microbiome, and consider evidence for the potentiating influence of moderate-to-vigorous physical activity (MVPA) combined with flavonoid intake. Improved cognition is associated with a physically active lifestyle, a healthy diet, and a robust, diverse gut microbiome (the gut-brain axis) (Schlegel et al., 2019; Westfall and Pasinetti, 2019). We and others have demonstrated that flavonoid-rich food interventions (such as cocoa, berries, or tea) can attenuate biomarkers of inflammation including neuroinflammation, effectively mitigate cognitive dysfunction and decline, and sharpen cognitive function (Macready et al., 2009; Whyte et al., 2020). Dietary flavonoids act as prebiotics; as such, they can alter the profiles and the diversity of the gut microbiome, and exercise accelerates the circulation and transport of flavonoid metabolites after gut microbiome catabolism. Here we review the extant literature and weave the three - flavonoids, exercise, and the gut microbiome together to form a more complete picture of how lifestyle affects cognition and how we can prevent the deterioration of cognitive abilities across the lifespan.

\section{FLAVONOIDS AND BRAIN HEALTH}

Flavonoids, a ubiquitous group of plant secondary metabolites with a 15-carbon structure (two phenyl rings and a heterocyclic ring; C6-C3-C6) are an indispensable component of traditional medicines, current nutraceuticals, and functional foods. Dietary flavonoids are found in tea, berryfruit, citrus and other fruit and legumes, although it is estimated that consumption in the United States falls well below dietary guidance (U.S. Department of Health and Human Services and U.S. Department of Agriculture, 2015). Over a decade ago, the potential for flavonoids to attenuate neurodegeneration was recognized (Spencer, 2009), and growing epidemiological, in vivo, and clinical evidence suggests that supplementation with flavonoid-rich foods benefits cognitive function (Letenneur et al., 2007; Dodd et al., 2019; Rajaram et al., 2019; Westfall and Pasinetti, 2019; Ruotolo et al., 2020; Whyte et al., 2020). In part, the underlying mechanisms may include flavonoids' anti-inflammatory capacity and influences on endothelial function and peripheral blood flow (Amin et al., 2015; Morais et al., 2016; Warner et al., 2017; Rajaram et al., 2019). We and others have established that dietary flavonoid metabolites pass the blood brain barrier and can be localized in brain tissues (Janle et al., 2010a,b;
Strathearn et al., 2014; Docampo et al., 2017; Angelino et al., 2019; Westfall and Pasinetti, 2019). Importantly, the flavonoid metabolites have been shown to deposit in brain regions that underlie learning and memory, specifically the hippocampus (Sokolov et al., 2013; Flanagan et al., 2018). Flavonoids are able to exert neuroprotective activity (even at the relatively low concentrations that reach the brain) by virtue of their ability to modulate protein and lipid kinase signaling pathways, and by inhibiting neuroinflammation, rather than merely through antioxidant activity. Absorbed flavonoids and their metabolites from foods (cocoa, berry, and tea) appear to directly interact with cellular and molecular targets (e.g., ERK and PI3-kinase/Akt signaling pathways) to improve neuronal connectivity and increase expression of neuromodulatory proteins (Williams and Spencer, 2012). Mechanistically, dietary flavonoid consumption promotes peripheral and cerebral vascular flow as well as neuronal survival and differentiation. Mounting evidence supports an association between flavonoid-rich plant-based diets and improved domains of cognition in aging, notably in executive functions, which are higher-order cognitive abilities subserved by the prefrontal lobe (Rajaram et al., 2019). These detriments in executive function begin to develop in midlife.

Preclinical and clinical studies with flavonoid-rich foods indicate that higher levels of flavonoid intake are related to improved cognitive performance and tempered cognitive decline (Macready et al., 2009; Hein et al., 2019; Whyte et al., 2020). Cocoa flavanol intake, for example, has been linked to greater brain oxygenation, and higher performance during cognitive challenge (Gratton et al., 2020), with apparent dose-dependent improvements in working memory, attention and processing speed (Socci et al., 2017). A systematic review concluded that green tea intake has a positive influence on cognition through the combined influence of green tea extract constituents, including flavonoids, L-theanine, and caffeine (Mancini et al., 2017). In preclinical experiments, however, it was administration of the green tea catechins (primarily EGCG) that were credited with improved spatial cognition learning ability in rats (improved reference and working memory) (Haque et al., 2006). Berries (a dietary resource with a highly diversified flavonoid profile), have been the intervention of choice for several trials on flavonoids and cognition (Galli et al., 2006; McGuire et al., 2006; Lau et al., 2007; Duffy et al., 2008; Williams et al., 2008; Small et al., 2014; Shukitt-Hale et al., 2015; Miller et al., 2018; Dodd et al., 2019; Whyte et al., 2020). Human clinical interventions with flavonoid subgroups anthocyanins, flavanol and flavanone over the past several years indicate potential to limit or reverse age-related declines in cognition (Ahles et al., 2021; Bird et al., 2021; Gardener et al., 2021). Blueberrysupplemented rats demonstrated elevated hippocampal levels of cAMP-response element-binding protein and extracellular signal-related kinase, and brain-derived neurotrophic factor (BDNF) compared to age-matched controls. Alteration of these signaling proteins led to better performance on a spatial working memory task (Williams et al., 2008; Vauzour et al., 2021). BDNF, known for differentiation and survival of neurons of the CNS, plays a crucial role in delay of cognitive aging by improving hippocampal plasticity, long-term memory, and neurogenesis 
(Cunha et al., 2010). Recently, clinical results showed that even a single acute dose of a flavonoid-rich blueberry beverage (equivalent to $200 \mathrm{~g}$ fresh berries; recognized as a reasonable, achievable dose) attenuated a decrease in plasma concentration of BDNF, whereas BDNF levels dropped in the placebo group (Dodd et al., 2019). Other gut-derived neuropeptides (GLP1, GLP-2, glucagon, etc.) affect brain activity, and can be modulated by flavonoid consumption (Badshah et al., 2013; $\mathrm{Gu}$ et al., 2014; Kashiwabara et al., 2016; Cremonini et al., 2021), suggesting another mechanism through which flavonoids enhance brain function.

The protective effects of flavonoid consumption occur primarily in the hippocampus, a brain area critical for memory function. A 3-month intervention with blueberry extract in non-impaired older adults showed significant improvements in delayed recognition and repetition errors (Whyte et al., 2018). Krikorian et al. (2010) investigated blueberry supplementation in older adults with mild cognitive impairment (MCI), finding robust improvement in a verbal paired-associate learning test. In a trial on working memory (WM), a significant increase in signaling in the left inferior parietal gyrus and left pre-central gyrus (enhanced neural activation) was found in older adults consuming blueberries, although behaviorally, performance on the WM task improved only marginally ( $p=0.08$ ) (Boespflug et al., 2018). More recently, a 24-week combined blueberry and/or fish oil intervention in older adults with cognitive deficits concluded that blueberry intervention improved cognitive efficiency for everyday life activities and resilience against extraneous disturbances during recognition memory tasks (McNamara et al., 2018).

In one of the longest duration blueberry interventions to date (Cheatham et al., 2022), we randomized older adults (aged 65-80) who were experiencing age-related cognitive changes to 6 months of wild blueberry or placebo. Participants who were not experiencing cognitive changes were included as a reference group. Participants were tested for cognitive abilities using the Montreal Cognitive Assessment (MoCA) (Nasreddine et al., 2005). Age-related cognitive change was operationalized as 1-1.5 SD below the standardized mean. Participants with lower scores
( $>1.5$ SD below the mean) were excluded from participation and referred to their physician. Cognitive abilities were gauged using the Cambridge Neuropsychological Test Automated Battery (CANTAB) and an electrophysiological technique known as event-related potentials (ERP). Those who consumed $35 \mathrm{~g}$ lyophilized blueberry powder/day (equivalent to $\sim 300 \mathrm{~g}$ or 2 cups fresh fruit) did not experience any further decline in abilities, whereas those on placebo did. In addition, those who consumed blueberries daily exhibited improvement in speed of processing (a basic cognitive ability that underlies all other cognitive abilities) (Figure 1, left). This improvement was evidenced in the behavioral tests (CANTAB) as well as the electrophysiological tests (ERP). In addition, recognition memory improved to the level of the reference group in the group consuming blueberries (Figure 1, right). That is, as measured in the ERP component $\mathrm{N} 2$, those consuming blueberries showed greater differentiation between processing (N2) in response to novel versus familiar stimuli relative to those consuming placebo. Thus, consumption of wild blueberries halted cognitive decline and improved speed of processing and recognition memory (Cheatham et al., 2022).

In sum, consumption of foods containing flavonoids appears to act in the brain to improve function (Letenneur et al., 2007; Ayaz et al., 2019). Flavonoids may enhance brain blood flow and block beta-amyloid plaque buildup (a hallmark of Alzheimer's disease) in the brain. As prevention is generally preferred and more readily achieved than remediation, we propose that consumption of flavonoid foods are central to the prevention of cognitive decline and quite possibly other brain functions such as executive function, attention, and memory (Socci et al., 2017; Westfall and Pasinetti, 2019).

\section{GUT MICROBIOME AND COGNITION}

The gut microbiome, an interactive community of microorganisms in the gastrointestinal tract, is highly influenced by diet (Westfall and Pasinetti, 2019; Zmora et al., 2019). Until very recently, only loosely attributed theories about the gastrointestinal microbial community's impact on brain function

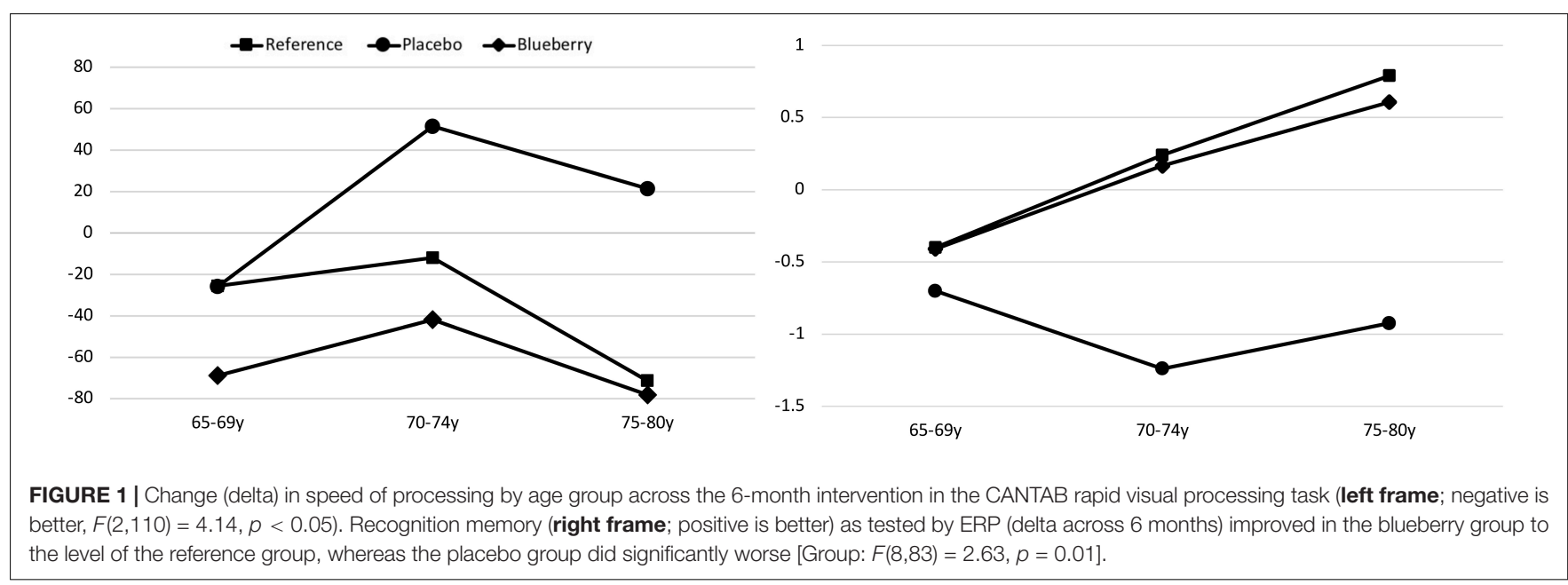


and behavior were available, based on highly controlled animal studies. Recent trials, however, have shown that institutionalized study participants experiencing cognitive decline have altered gut bacterial composition compared to participants with typical cognitive ability (Scheperjans et al., 2015; Bajaj et al., 2016). These studies imply that the composition and diversity of gut microbiota may significantly modulate gut-brain communication, contributing to changes in cognition during aging (Anderson et al., 2017; Manderino et al., 2017). It has even been suggested that "westernization" of lifestyles, including western diets and the habitual use of antibiotic treatments which disrupt the gut microbiome, may contribute to neurological dysfunction (Novotny et al., 2019).

A comparative review of almost a score of recent human clinical trials suggested that deliberate intervention to change gut microbiota composition can produce a positive bacteriacognition relationship, eliciting improvements in visuospatial memory, verbal learning, and attention (Tooley, 2020). In this narrative review, the importance of recognizing microbiota signatures associated with cognitive performance, and identifying potential gut microbiota interventions (including diet and lifestyle) were highlighted. In another clinical trial, an increased prevalence of Bacteroides in the gut microbiome was associated with mild cognitive impairment in geriatric patients, evidenced by impaired memory and lower global cognitive function scores (Saji et al., 2019). The gut microbiota-brain axis has previously been implicated in development of neurological diseases including Alzheimer's disease, and is proposed as a target for cognitive decline therapeutics (Borsom et al., 2020). Deliberate modulation of gut microbiome profiles, either by fecal transplantation or probiotic interventions, is gaining research momentum in the quest to control the pathogenesis of Alzheimer's disease (Wang and Dykes, 2021).

The microbiota-gut-brain communication is bidirectional; changes in the composition of the gut microbiota are associated with behavioral and cognitive alternations, and, perturbations in behavior also alter the composition of the gut microbiota (Mu et al., 2016; Borsom et al., 2020). Gut microbial metabolites (including postbiotics such as bile acids, short chain fatty acids, and tryptophan metabolites, as well as phenolic metabolites from flavonoid catabolism) are major mediators of the microbiomegut-brain axis (Cryan and Dinan, 2012; Banfi et al., 2021). We posit that cognition (memory and executive function) are linked to the composition, functionality, and diversity of the gut microbiota, in part because gut microbiota regulate the production and delivery of microbiome-catabolized phenolic metabolites into circulation following intake of flavonoid-rich foods (Dinan et al., 2015; Manderino et al., 2017; Chu et al., 2019).

Using clinically validated behavioral measures and electrophysiological measures of brain activity on a cohort of older adults between 67 and 83 years of age and a diversity metric for the gut microbiome, we recently found that gut microbial diversity, as modulated by diet/flavonoid intake, was a predictor of cognitive performance in free-living aging adults (Canipe et al., 2021). We saw a significant association between behavioral measures of paired-associate learning and spatial working memory, and the $\alpha$-diversity of the gut microbiome of older adults; poorer performance (indicative of cognitive dysfunction) predicted lower gut microbiome diversity (Canipe et al., 2021). Poorer performance on spatial working memory tests and paired associates learning was related to lower Shannon $\alpha$-scores (a diversity metric) (Peet, 1974) in the gut microbiome (Figure 2). Electrophysiology waveforms related to attention differentially predicted gut microbiome diversity, such that those with better attention allocation and better sustained attention abilities had more diverse gut microbiomes. Thus, higher gut microbiome diversity is related to better brain function as measured by cognitive tests. Importantly, in this same sample, we related free-living consumption of berries to the diversity score and found that those who reported eating more servings of berries across three 24-h diet recalls had a more diverse gut microbiome) (Canipe et al., 2021). Interacting ingested flavonoids can serve a pivotal role in changing or reshaping the gut microbiota, increasing populations of Lactobacilli spp. and Bifidobacteria spp. and inhibiting gut pathogens (Wang et al., 2021). Reduced abundance of pathogenic bacteria in the gut (Clostridium perfringens, C. difficile, and gram-negative Bacteroides spp.) without inhibition of commensal bacteria (clostridia and lactobacilli) has been linked to prebiotic polyphenol intake (Lee et al., 2006; Tuohy et al., 2012; Duda-Chodak et al., 2015). Separate in vivo feeding trials with berry species (cranberry and grape) led to consistent decrease in proportion of Firmicutes to Bacteroidetes, and remarkable increase in growth of Akkermansia muciniphila in the microbial community (Anhe et al., 2014; Roopchand et al., 2015; Wang et al., 2021).

In sum, consumption of flavonoids is related to improved speed of processing and recognition memory; consumption of berry flavonoids is related to a more highly diverse gut microbiome; and higher $\alpha$-diversity in the gut microbiome is related to better cognitive abilities (Canipe et al., 2021). The next section will further explore how flavonoid consumption alters the gut microbiome.

\section{FLAVONOIDS AND THE GUT MICROBIOME}

Interactions between the microbiome and dietary flavonoids have dual impacts on human health. First, diet, including prebiotic flavonoid-rich foods, has a dramatic influence on the composition and consequently the functionality of the gut microbiome. The term prebiotic usually refers to indigestible dietary fibers that benefit the gut microbiota, and flavonoids are considered to have prebiotic-like effects as their consumption also feeds/benefits commensal bacteria in the gut. Ingesting prebiotics alters gut microbial community structure, favoring beneficial commensal bacteria, and reducing levels of opportunistic species (Anhe et al., 2014; Roopchand et al., 2015; Westfall et al., 2018). Prebiotic flavonoids' metabolites also have a profound influence on inflammation in the gut, improving the epithelial barrier's integrity and activating tight junctions (Anhe et al., 2014; Westfall and Pasinetti, 2019). Interestingly, the flavonoids do not even need to be absorbed to exert these benefits; as xenobiotic compounds, they induce cellular stress and an overcompensation 
reaction to maintain homeostasis, producing a hormetic response that improves cell and barrier function (Calabrese et al., 2012). The same gut-derived flavonoid metabolites that suppress chronic intestinal inflammation are integral to inhibition of neuroinflammation (Spencer et al., 2012).

In tandem, the gut microbiome significantly impacts flavonoid bioavailability via extensive pre-systemic metabolism to release active metabolites with therapeutic efficacy, including for cognitive benefits. Only a small percentage of dietary flavonoids are absorbed from the small intestine, before they reach the colonic microbiota. Instead, most dietary flavonoids are biotransformed by commensal gut microbiota into diverse bioactive phenolic metabolites, and are delivered into circulation where they elicit health-protective effects (Jennings et al., 2019). The bioavailability of flavonoids is therefore largely dependent on their catabolism by the gut microbiome and subsequent secondary xenobiotic biotransformation in the liver before entering circulation in the form of phenolic metabolites (Espin et al., 2017; Williamson et al., 2018; Westfall and Pasinetti, 2019). Thus, flavonoid bioavailability depends, in part, on the polyphenol-microbiota interactions, which ultimately regulate both bioavailability and bioactivity (Lila et al., 2016).

Extensive evidence indicates that biotransformed metabolites from lower-intestinal bacterial catabolism of dietary flavonoids mediate anti-inflammatory activity in multiple tissues of the body (Moco et al., 2012; Morais et al., 2016; Tomas-Barberan et al., 2016; Schell et al., 2019; Deledda et al., 2021; Martin and Ramos, 2021). Notably, diseases which cause chronic lowgrade inflammation (metabolic syndrome, diabetes, and arthritis) are strongly linked to cognitive decline in aging (Santoro et al., 2014; Noble et al., 2017). Strategies to attenuate the chronic, low grade inflammatory status characteristic of aging adults (inflammaging) can evoke systemic benefits on both physical and cognitive health. The gut microbiota is also essential for producing the full battery of bioavailable plasma- and brain-bioactive metabolites that have neuroprotective capacity
(Westfall and Pasinetti, 2019). Long-term supplementation with a probiotic could promote health by introducing colonic microbiota that make flavonoids more bioavailable (Pereira-Caro et al., 2015; Westfall and Pasinetti, 2019).

Evidence from our team and others shows that gut microbialderived metabolites of dietary flavonoids are anti-inflammatory and immunomodulatory, can have greater bioactivity than their parent/precursor structures, and have additive or synergistic effects collectively (Ahmed et al., 2014; Amin et al., 2015; DudaChodak et al., 2015; Nieman et al., 2017, 2018; Warner et al., 2017). Indeed, daily consumption of anthocyanins in a dietinduced obese mouse model with either healthy or antibioticdisrupted gut microbiota resulted in reduced body weight gain and improved glucose metabolism, but only in mice with intact gut microbiota (Esposito et al., 2015). The bidirectional breakdown of flavonoids into active and more bioaccessible metabolites and concurrent modulation of the gut microbial community by these metabolites, both contribute to positive health outcomes.

So far, we have described the positive associations between flavonoid ingestion and cognitive health; the connections between a robust microbiome and cognitive health; and the two-way interactions between the gut microbiome and ingested flavonoids, which mediate both the potency and bioavailability of flavonoid metabolites; and the composition and functionality of the microbiome. How might lifestyle factors, aside from diet, have a bearing on these cognition-relevant influences?

\section{PHYSICAL ACTIVITY AS A MEDIATOR}

A physically active lifestyle is intrinsically linked to brain health. Physically active people are less likely to demonstrate cognitive decline, all-cause dementia, vascular dementia, and Alzheimer's disease; most data support MVPA for at least 150 min per week (Middleton et al., 2008; Voss et al., 2011;
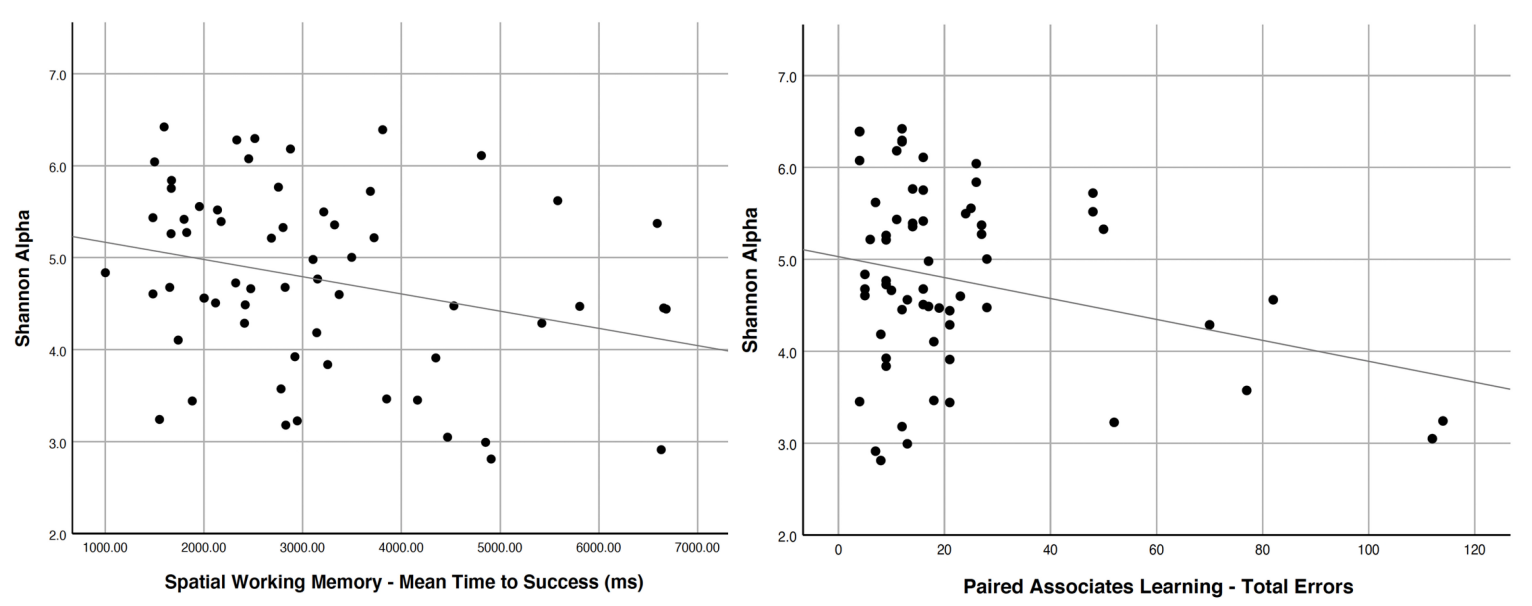

FIGURE 2 | Cognitive scores (CANTAB) related to the gut microbiome diversity score Shannon Alpha [Multivariate Model $\left.F(2,56)=4.846, R^{2}=0.117\right]$ on spatial working memory (left-longer times to success relate to lower diversity; $p=0.025$ ) and paired associates learning (right-higher errors on the task relate to lower diversity; $p=0.024)$. Reprinted from Canipe et al. (2021). 
Gow et al., 2012; Napoli et al., 2014; Piercy and Troiano, 2018; Minghui, 2019; Chen et al., 2020). MVPA for elderly adults has benefits for cognitive performance, brain function, and brain structure (Voss et al., 2011). Most meta-analyses and systematic reviews support that regular MVPA improves various aspects of cognitive function including executive function (EF), language ability, visuospatial ability, and memory in older adults with cognitive impairment (Chen et al., 2020; Zhou et al., 2020). When physical activity was directly monitored, participants engaging in recommended levels of MVPA had lower incidence of cognitive impairment and better maintenance of EF and memory (Zhu et al., 2016).

The Physical Activity Guidelines for Americans state that some benefits of physical activity on cognitive health occur immediately after a session of MVPA (acute effect); these include reduced anxiety, improved sleep, and improved cognitive function (Piercy and Troiano, 2018). With regular MVPA by older adults with or without impaired cognitive health (chronic effect), even greater cognitive benefits are experienced including improvements in EF, attention, memory, crystallized intelligence, and processing speed (Weng et al., 2015; Tsukamoto et al., 2017; Piercy and Troiano, 2018). The substantial cognitive benefits observed with at least 150 min per week MVPA are further amplified when MVPA is increased to 300 min per week. Potential mechanisms for MVPA's positive impacts on cognition include changes in brain structures (Rovio et al., 2010; Devenney et al., 2017), increases in cerebral blood flow and oxygentation (Rooks et al., 2010; Tsubaki et al., 2021), enhanced immune function, reductions in inflammation including neuroinflammation, and/or increase neurotrophic factors (Nieman et al., 2013; Ahmed et al., 2014; Amin et al., 2015; Rehfeld et al., 2018; Minghui, 2019). Habitual walking in late adulthood has been correlated with higher gray matter volume, coincident with reduced risk of cognitive dysfunction (Erickson et al., 2010). Flavonoid benefits coincident with physical exertion are in part due to antioxidant and antiinflammatory effects, but also since these polyphenols activate the same adaptive cell signaling pathways as physical exertion, they are believed to complement adaptive benefits of exercise and support performance (Hurst et al., 2019).

Moderate-to-vigorous physical activity combined with flavonoid ingestion may improve post-exercise metabolic recovery (Hurst et al., 2019; Nieman et al., 2019, 2020), and augment cognitive function (Tsukamoto et al., 2018). Effects of MVPA and flavonoid ingestion may be mediated in part through elevations in circulating gut-derived phenolic metabolites, but this linkage has not yet been conclusively established (Nieman et al., 2013, 2018). MVPA does enhance the release of gut-derived phenolic metabolites following chronic flavonoid ingestion. In a randomized trial with long-distance runners featuring a 17-day intervention with a flavonoid-rich supplement (or placebo), serum metabolic signatures from colonic flavonoid metabolites (derived from green tea or berries) were significantly elevated for at least $14 \mathrm{~h}$ coincident with a 3-day intensified exercise period, and these changes persisted through postexercise recovery (Nieman et al., 2013). Microbial metabolites were dramatically elevated once the workout commenced the release of the metabolites into plasma was stimulated by

Flavonoid-Run

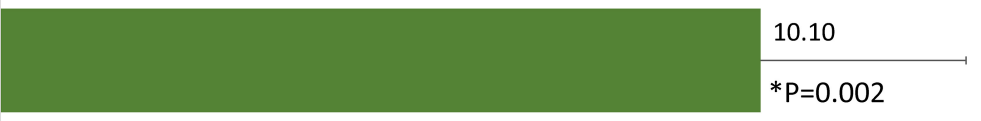

Flavonoid-Walk

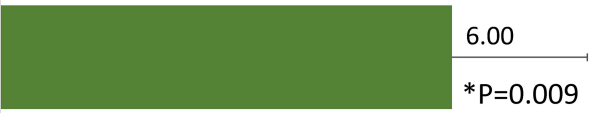

Flavonoid-Sit

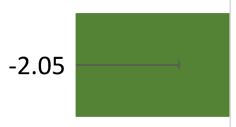

Placebo-Walk

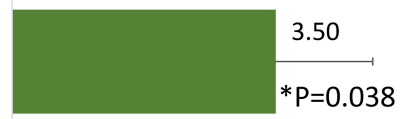

Placebo-Sit

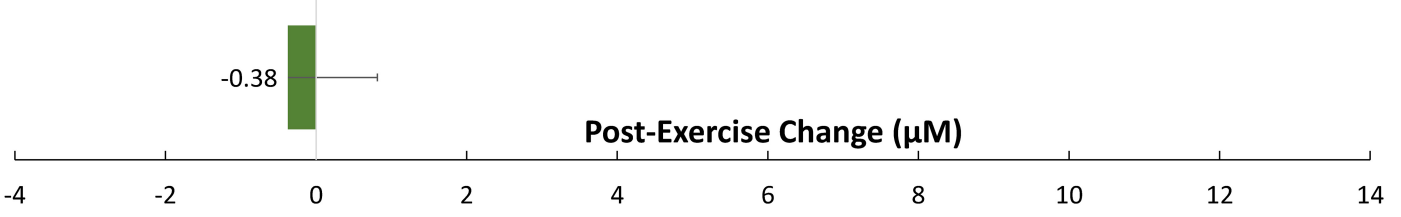

FIGURE 3 | Post-exercise change for 15 selected and grouped plasma gut-derived phenolics. P-values indicate contrasts with the placebo-sit group. Data are represented as means with standard errors of the mean. *indicates a significant difference with the placebo-sit group $(P$ values). 
physical exertion. Release of these microbial metabolites into circulation significantly countered the athletes' typical postexercise susceptibility to virus infection, by depressing ex vivo viral replication and attenuating virulence (Ahmed et al., 2014).

In another study, the combination of 2 weeks of flavonoid supplementation and acute exercise (both 45 min brisk walking and $2.5 \mathrm{~h}$ running) enhanced the translocation of gut-derived phenolics into circulation (Figure 3; Nieman et al., 2018). The pre-study plasma concentration of gut-derived phenolic metabolites was $40 \%$ higher in the leaner and fitter runners than in the walkers. These data indicated that acute exercise bouts (both brisk walking and intensive running) combined with flavonoid supplementation, and an elevated fitness status associated with habitual running, were linked to elevations in plasma levels of gut-derived phenolics.

Several underlying mechanisms could explain these results including flavonoid- and exercise-induced changes in gut permeability and transporter function, increases in gut microbiota richness, and altered gastrointestinal motility and transport rate (Nieman et al., 2018). Chronic MVPA can modify the composition and functional capacity of the gut microbiota (Hughes and Holscher, 2021). Cross-sectional human studies have revealed greater $\alpha$-diversity and an enriched profile of short chain fatty acids (SCFAs) in athletes compared to sedentary controls (Barton et al., 2018; Mailing et al., 2019; Nieman and Pence, 2020; Zhu et al., 2020). One examination of phenotypic features across 3,400 individuals found a linkage between microbiome diversity and MVPA frequency and duration that was independent of major dietary

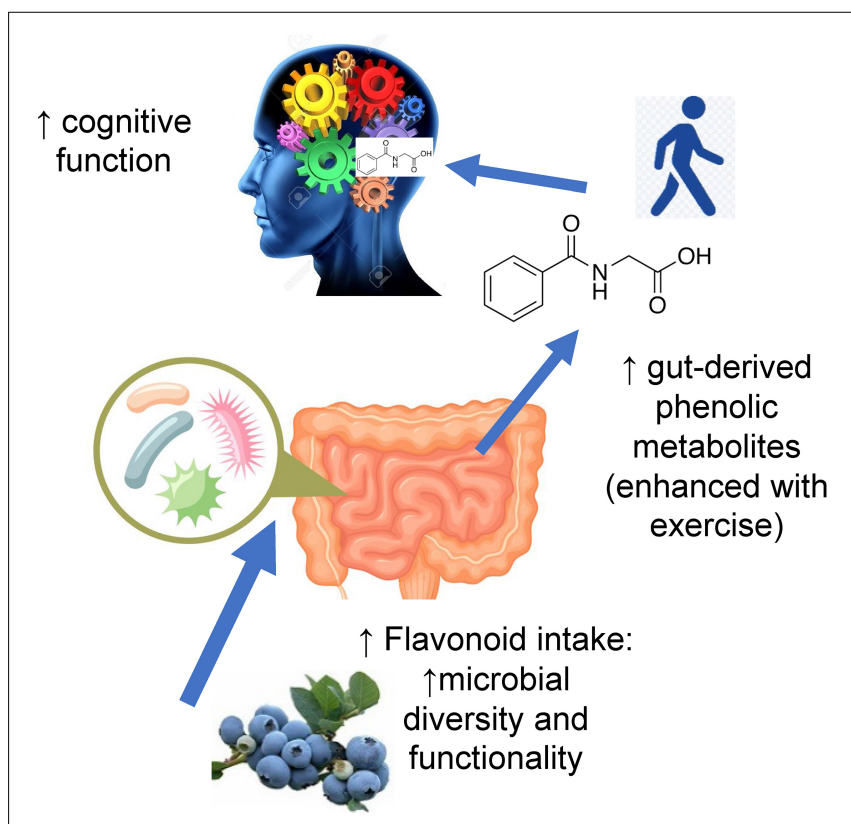

FIGURE 4 | Higher flavonoid intake may influence cognitive function by augmenting gut microbial diversity and functionality and increasing circulating levels of gut-derived phenolic metabolites. Moderate-to-vigorous physical activity (MVPA) adds to this effect by enhancing the release of gut-derived metabolites and improving cognitive function. factors and BMI (Manor et al., 2020) and longitudinal MVPA studies support some selective changes on the gut microbiome, especially when vigorous exercise training is sustained for months (Allen et al., 2018; Cronin et al., 2018; Kern et al., 2020). The interaction between MVPA and the gut microbiota is bidirectional. As already noted, flavonoids are catabolized by and influence composition of the microbiota, and exercise stimulated the bioavailability of flavonoid metabolites (Nieman et al., 2018). The gut microbiota also has an influence on exercise performance by producing SCFAs that increase muscle blood flow and insulin sensitivity, and can be utilized as fuel (Hughes and Holscher, 2021).

It is therefore reasonable to posit that the connection between physical activity (e.g., exercise), dietary flavonoids, and improved cognition (memory and executive function) is linked to their demonstrated influence on the microbiome (diversity and functionality) (Figure 4).

\section{CONCLUSION}

A complex interaction of factors (lifestyle, diet, genetics, and environment) all appear to exert influence on cognition in the aging brain. Emerging evidence suggests that there may be potentiating interactions between some of these, including flavonoid intake, microbiome, and physical activity levels (exercise). What are the mechanisms responsible for the benefits to cognition, and how can they be fully demonstrated? We hypothesize that it is the phytoactive metabolites from flavonoid ingestion, after catabolism at the gut microbiome level, that interact with cellular and molecular targets (signaling pathways) to improve neuron connectivity and promote vascular and peripheral flow in the brain. Exercise has demonstrated ability to provoke a surge of these phytoactive flavonoid metabolites into circulation. It follows that the positive cognitive benefits from dietary flavonoids and regular moderate exercise may be a consequence of the enhanced circulation of gut-derived flavonoid metabolites, mediated by the activities of the colonic microbiota.

\section{AUTHOR CONTRIBUTIONS}

CC: cognition. DN: physical movement. AN: microbiome. ML: flavonoids/metabolites. All authors contributed to and approved the manuscript.

\section{FUNDING}

Some of the work described in this review was partially funded through USDA-ARS Project Nos. 0204-41510-001-24S and USDA NIFA Hatch Project 02689.

\section{ACKNOWLEDGMENTS}

First and foremost, we thank the generous people who participate in our research. Without them, our research would literally be impossible. 


\section{REFERENCES}

Ahles, S., Joris, P. J., and Plat, J. (2021). Effects of berry anthocyanins on cognitive performance, vascular function and cardiometabolic risk markers: a systematic review of randomized placebo-controlled intervention studies in humans. Int. J. Mol. Sci. 22:6482. doi: 10.3390/ijms22126482

Ahmed, M., Henson, D. A., Sanderson, M. C., Nieman, D. C., Gillitt, N. D., and Lila, M. A. (2014). The protective effects of a polyphenol-enriched protein powder on exercise-induced susceptibility to virus infection. Phytother. Res. 28, 1829-1836. doi: $10.1002 /$ ptr.5208

Allen, J. M., Mailing, L. J., Niemiro, G. M., Moore, R., Cook, M. D., White, B. A., et al. (2018). Exercise alters gut microbiota composition and function in lean and obese humans. Med. Sci. Sports Exerc. 50, 747-757. doi: 10.1249/MSS. 0000000000001495

Amin, H. P., Czank, C., Raheem, S., Zhang, Q., Botting, N. P., Cassidy, A., et al. (2015). Anthocyanins and their physiologically relevant metabolites alter the expression of IL-6 and VCAM-1 in CD40L and oxidized LDL challenged vascular endothelial cells. Mol. Nutr. Food Res. 59, 1095-1106. doi: 10.1002/ mnfr. 201400803

Anderson, J. R., Carroll, I., Azcarate-Peril, M. A., Rochette, A. D., Heinberg, L. J., Peat, C., et al. (2017). A preliminary examination of gut microbiota, sleep, and cognitive flexibility in healthy older adults. Sleep Med. 38, 104-107.

Angelino, D. C., Carregosa, D., Domenech-Coca, C., Savi, M., Figueira, I., Brindani, N., et al. (2019). 5-(hydroxyphenyl)-valerolactone-sulfate, a key microbial metabolite of flavan-3-ols, is able to reach the brain: evidence from different in silico, in vitro and in vivo experimental models. Nutrients 11:2678.

Anhe, F. F., Roy, D., Pilon, G., Dudonné, S., Matamoros, S., Varin, T. V., et al. (2014). A polyphenol-rich cranberry extract protects from diet-induced obesity, insulin resistance and intestinal inflammation in association with increased Akkermansia spp. population in the gut microbiota of mice. Gut 64, 872-883.

Ayaz, M., Sadiq, A., Junaid, M., Ullah, F., Ovais, M., Ullah, I., et al. (2019). Flavonoids as prospective neuroprotectants and their therapeutic propensity in aging associated neurological disorders. Front. Aging Neurosci. 11:155. doi: 10.3389/fnagi.2019.00155

Badshah, H., Ullah, I., Kim, S. E., Kim, T. H., Lee, H. Y., and Kim, M. O. (2013). Anthocyanins attenuate body weight gain via modulating neuropeptide $\mathrm{Y}$ and GABAB1 receptor in rats hypothalamus. Neuropeptides 47, 347-353. doi: 10. 1016/j.npep.2013.06.001

Bajaj, J. S., Ahluwalia, V., Steinberg, J. L., Hobgood, S., Boling, P. A., Godschalk, M., et al. (2016). Elderly patients have an altered gut-brain axis regardless of the presence of cirrhosis. Sci. Rep. 6:38481. doi: 10.1038/srep38481

Banfi, D., Moro, E., Bosi, A., Bistoletti, M., Cerantola, S., Crema, F., et al. (2021). Impact of microbial metabolites on microbiota-gut-brain axis in inflammatory bowel disease. Int. J. Mol. Sci. 22:1623. doi: 10.3390/ijms22041623

Barton, W., Penney, N. C., Cronin, O., Garcia-Perez, I., Molloy, M. G., Holmes, E., et al. (2018). The microbiome of professional athletes differs from that of more sedentary subjects in composition and particularly at the functional metabolic level. Gut 67, 625-633. doi: 10.1136/gutjnl-2016-313627

Bird, R. J., Hoggard, N., and Aceves-Martins, M. (2021). The effect of grape interventions on cognitive and mental performance in healthy participants and those with mild cognitive impairment: a systematic review of randomized controlled trials. Nutr. Rev. nuab025. doi: 10.1093/nutrit/nuab025

Boespflug, E. L., Eliassen, J. C., Dudley, J. A., Shidler, M. D., Kalt, W., Summer, S. S., et al. (2018). Enhanced neural activation with blueberry supplementation in mild cognitive impairment. Nutr. Neurosci. 21, 297-305. doi: 10.1080/ 1028415X.2017.1287833

Borsom, E. M., Lee, K., and Cope, E. K. (2020). Do the bugs in your gut eat your memories? relationship between gut microbiota and Alzheimer.s disease. Brain Sci. 10:814. doi: 10.3390/brainsci10110814

Calabrese, V., Cornelius, C., Dinkova-Kostova, A. T., Iavicoli, I., Di Paola, R., Koverech, A., et al. (2012). Cellular stress responses, hormetic phytochemicals and vitagenes in aging and longevity. Biochim. Biophys. Acta 1822, 753-783. doi: 10.1016/j.bbadis.2011.11.002

Canipe, L. G., Sioda, M., and Cheatham, C. L. (2021). Diversity of the gutmicrobiome related to cognitive behavioral outcomes in healthy older adults. Arch. Gerontol. Geriatr. 96:104464. doi: 10.1016/j.archger.2021.104464

Cheatham, C., Millsap, G., Chai, S. C., Canipe, G., Sheppard, K. W., and Lila, M. A. (2022). Consumption of wild blueberries for 6 months improves speed of processing in 65 - to 80 -year-olds experiencing mild cognitive decline. Am. J. Clin. Nutr.

Chen, F. T., Etnier, J. L., Chan, K. H., Chiu, P. K., Hung, T. M., and Chang, Y. K. (2020). Effects of exercise training interventions on executive function in older adults: a systematic review and meta-analysis. Sports Med. 50, 1451-1467. doi: 10.1007/s40279-020-01292-x

Chu, C., Murdock, M. H., Jing, D., Won, T. H., Chung, H., Kressel, A. M., et al. (2019). The microbiota regulate neuronal function and fear extinction learning. Nature 574, 543-548. doi: 10.1038/s41586-019-1644-y

Cremonini, E., Daveri, E., Mastaloudis, A., and Oteiza, P. I. (2021). (-)-Epicatechin and anthocyanins modulate GLP-1 metabolism: evidence from C57BL/6J mice and GLUTag cells. J. Nutr. 151, 1497-1506. doi: 10.1093/jn/nxab029

Cronin, O., Barton, W., Skuse, P., Penney, N. C., Garcia-Perez, I., Murphy, E. F., et al. (2018). A prospective metagenomic and metabolomic analysis of the impact of exercise and/or whey protein supplementation on the gut microbiome of sedentary adults. mSystems 3:e00044-18. doi: 10.1128/mSystems.00 044-18

Cryan, J. F., and Dinan, T. G. (2012). Mind-altering microorganisms: the impact of the gut microbiota on brain and behaviour. Nat. Rev. Neurosci. 13, 701-712. doi: $10.1038 / \mathrm{nrn} 3346$

Cunha, C., Brambilla, R., and Thomas, K. (2010). A simple role for BDNF in learning and memory? Front. Mol. Neurosci. 3:1. doi: 10.3389/neuro.02.001. 2010

Deledda, A., Annunziata, G., Tenore, G. C., Palmas, V., Manzin, A., and Velluzzi, F. (2021). Diet-derived antioxidants and their role in inflammation, obesity and gut microbiota modulation. Antioxidants 10:708. doi: 10.3390/antiox10050708

Devenney, K. E., Sanders, M. L., Lawlor, B., Olde Rikkert, M. G. M., Schneider, S., and NeuroExercise Study Group (2017). The effects of an extensive exercise programme on the progression of mild cognitive impairment (MCI): study protocol for a randomised controlled trial. BMC Geriatr. 17:75. doi: 10.1186/ s12877-017-0457-9

Dinan, T. G., Stilling, R. M., Stanton, C., and Cryan, J. F. (2015). Collective unconscious: how gut microbes shape human behavior. J. Psychiatr. Res. 63, 1-9. doi: 10.1016/j.jpsychires.2015.02.021

Docampo, M., Olubu, A., Wang, X., Pasinetti, G., and Dixon, R. A. (2017). Glucuronidated flavonoids in neurological protection: structural analysis and approaches for chemical and biological synthesis. J. Agric. Food Chem. 65, 7607-7623. doi: 10.1021/acs.jafc.7b02633

Dodd, G. F., Williams, C. M., Butler, L. T., and Spencer, J. P. E. (2019). Acute effects of flavonoid-rich blueberry on cognitive and vascular function in healthy older adults. Nutr. Heal. Aging 5, 119-132.

Duda-Chodak, A., Tarko, T., Satora, P., and Sroka, P. (2015). Interaction of dietary compounds, especially polyphenols, with the intestinal microbiota: a review. Eur. J. Nutr. 54, 325-341. doi: 10.1007/s00394-015-0852-y

Duffy, K. B., Spangler, E. L., Devan, B. D., Guo, Z., Bowker, J. L., Janas, A. M., et al. (2008). A blueberry-enriched diet provides cellular protection against oxidative stress and reduces a kainate-induced learning impairment in rats. Neurobiol. Aging 29, 1680-1689.

Erickson, K. I., Raji, C. A., Lopez, O. L., Becker, J. T., Rosano, C., Newman, A. B., et al. (2010). Physical activity predicts gray matter volume in late adulthood: the cardiovascular health study. Neurology $75,1415-1422$. doi: 10.1212/WNL. 0b013e3181f88359

Espin, J. C., Gonzalez-Sarrias, A., and Tomas-Barberan, F. (2017). The gut microbiota: a key factor in the therapeutic effects of (poly)phenols. Biochem. Pharmacol. 139, 82-93.

Esposito, D., Damsud, T., Wilson, M., Grace, M. H., Strauch, R., Li, X., et al. (2015). Black currant anthocyanins attenuate weight gain and improve glucose metabolism in diet-induced obese mice with intact, but not disrupted, gut microbiome. J. Agric. Food Chem. 63, 6172-6180. doi: 10.1021/acs.jafc.5b00963

Flanagan, E., Muller, M., Hornberger, M., and Vauzour, D. (2018). Impact of flavonoids on cellular and molecular mechanisms underlying age-related cognitive decline and neurodegeneration. Curr. Nutr. Rep. 7, 49-57. doi: 10. 1007/s13668-018-0226-1

Galli, R. L., Bielinski, D. F., Szprengiel, A., Shukitt-Hale, B., and Joseph, J. A. (2006). Blueberry supplemented diet reverses age-related decline in hippocampal HSP70 neuroprotection. Neurobiol. Aging 27, 344-350.

Gardener, S. L., Rainey-Smith, S. R., Weinborn, M., Bondonno, C. P., and Martins, R. N. (2021). Intake of products containing anthocyanins, flavanols, and 
flavanones, and cognitive function: a narrative review. Front. Aging Neurosci. 13:640381. doi: 10.3389/fnagi.2021.640381

Gow, A. J., Mortensen, E. L., and Avlund, K. (2012). Activity participation and cognitive aging from age 50 to 80 in the Glostrup 1914 cohort. J. Am. Geriatr. Soc. 60, 1831-1838. doi: 10.1111/j.1532-5415.2012.04168.x

Gratton, G., Weaver, S. R., Burley, C. V., Low, K. A., Maclin, E. L., Johns, P. W., et al. (2020). ). Dietary flavanols improve cerebral cortical oxygenation and cognition in healthy adults. Sci. Rep. 10, 19409-19409. doi: 10.1038/s41598-020-76160-9

Gu, Y., Yu, S., Park, J. Y., Harvatine, K., and Lambert, J. D. (2014). Dietary cocoa reduces metabolic endotoxemia and adipose tissue inflammation in high-fat fed mice. J. Nutr. Biochem. 25, 439-445. doi: 10.1016/j.jnutbio.2013.12.004

Haque, A. M., Hashimoto, M., Katakura, M., Tanabe, Y., Hara, Y., and Shido, O. (2006). Long-term administration of green tea catechins improves spatial cognition learning ability in rats. J. Nutr. 136, 1043-1047.

Hein, S., Whyte, A. R., Wood, E., Rodriguez-Mateos, A., and Williams, C. M. (2019). Systematic review of the effects of blueberry on cognitive performance as we age. J. Gerontol. A Biol. Sci. Med. Sci. 74, 984-995. doi: 10.1093/gerona/ glz082

Hughes, R. L., and Holscher, H. D. (2021). Fueling gut microbes: a review of the interaction between diet, exercise, and the gut microbiota in athletes. Adv. Nutr. $12,2190-2215$.

Hurst, R. D., Lyall, K. A., Roberts, J. M., Perthaner, A., Wells, R. W., Cooney, J. M., et al. (2019). Consumption of an anthocyanin-rich extract made from New Zealand blackcurrants prior to exercise may assist recovery from oxidative stress and maintains circulating neutrophil function: a pilot study. Front. Nutr. 6:73. doi: $10.3389 /$ fnut. 2019.00073

Janle, E. M., Lila, M. A., Grannan, M., Wood, L., Higgins, A., Yousef, G. G., et al. (2010a). Method for evaluating the potential of $\mathrm{C}^{14}$ labeled plant polyphenols to cross the blood-brain barrier using accelerator mass spectrometry. Nucl. Instrum. Methods Phys. Res. B 268, 1313-1316. doi: 10.1016/j.nimb.2009.10.161

Janle, E. M., Lila, M. A., Grannan, M., Wood, L., Higgins, A., Yousef, G. G., et al. (2010b). Pharmacokinetics and tissue distribution of 14C-labeled grape polyphenols in the periphery and the central nervous system following oral administration. J. Med. Food 13, 926-933. doi: 10.1089/jmf.2009.0157

Jennings, A., Koch, M., Jensen, M. K., Bang, C., Kassubek, J., Müller, H. P., et al. (2019). The role of the gut microbiome in the association between habitual anthocyanin intake and visceral abdominal fat in population-level analysis. Am. J. Clin. Nutr. 111, 340-350

Kashiwabara, M., Asano, K., Mizuyoshi, T., and Kobayashi, H. (2016). Suppression of neuropeptide production by quercetin in allergic rhinitis model rats. $B M C$ Complement Altern. Med. 16:132. doi: 10.1186/s12906-016-1123-z

Kern, T., Blond, M. B., Hansen, T. H., Rosenkilde, M., Quist, J. S., Gram, A. S., et al. (2020). Structured exercise alters the gut microbiota in humans with overweight and obesity-A randomized controlled trial. Int. J. Obes 44, 125-135. doi: 10.1038/s41366-019-0440-y

Krikorian, R., Shidler, M. D., Nash, T. A., Kalt, W., Vinqvist-Tymchuk, M. R., Shukitt-Hale, B., et al. (2010). Blueberry supplementation improves memory in older adults. J. Agric. Food Chem. 58, 3996-4000. doi: 10.1021/jf9029332

Lau, F. C., Bielinski, D. F., and Joseph, J. A. (2007). Inhibitory effects of blueberry extract on the production of inflammatory mediators in lipopolysaccharideactivated BV2 miroglia. J. Neurosci. Res. 85, 1010-1017. doi: 10.1002/jnr.21205

Lee, H. C., Jenner, A. M., Low, C. S., and Lee, Y. K. (2006). Effect of tea phenolics and their aromatic fecal bacterial metabolites on intestinal microbiota. Res. Microbiol. 157, 876-884. doi: 10.1016/j.resmic.2006.07.004

Letenneur, L., Proust-Lima, C., Le Gouge, A., Dartigues, J., and Barberger-Gateau, P. (2007). Flavonoid intake and cognitive decline over a 10-year period. Am. J. Epidemiol. 165, 1364-1371. doi: 10.1093/aje/kwm036

Lila, M. A., Burton-Freeman, B., Grace, M., and Kalt, W. (2016). Unraveling anthocyanin bioavailability for human health. Annu. Rev. Food Sci. Technol. 7, 375-393. doi: 10.1146/annurev-food-041715-033346

Macready, A. L., Kennedy, O. B., Ellis, J. A., Williams, C. M., Spencer, J. P., and Butler, L. T. (2009). Flavonoids and cognitive function: a review of human randomized controlled trial studies and recommendations for future studies. Genes Nutr. 4, 227-242. doi: 10.1007/s12263-009-0135-4

Mailing, L. J., Allen, J. M., Buford, T. W., Fields, C. J., and Woods, J. A. (2019). Exercise and the gut microbiome: a review of the evidence, potential mechanisms, and implications for human health. Exerc. Sport Sci. Rev. 47, 75-85. doi: 10.1249/JES.0000000000000183
Mancini, E., Beglinger, C., Drewe, J., Zanchi, D., Lang, U. E., and Borgwardt, S. (2017). Green tea effects on cognition, mood and human brain function: a systematic review. Phytomedicine 34, 26-37.

Manderino, L., Carroll, I., Azcarate-Peril, M. A., Rochette, A., Heinberg, L., Peat, C., et al. (2017). Preliminary evidence for an association between the composition of the gut microbiome and cognitive function in neurologically healthy older adults. J. Int. Neuropsychol. Soc. 23, 700-705. doi: 10.1017/ S1355617717000492

Manor, O., Dai, C. L., Kornilov, S. A., Smith, B., Price, N. D., Lovejoy, J., et al. (2020). Health and disease markers correlate with gut microbiome composition across thousands of people. Nat. Commun. 11:5206. doi: 10.1038/s41467-02018871-1

Martin, M. A., and Ramos, S. (2021). Impact of dietary flavanols on microbiota, immunity and inflammation in metabolic diseases. Nutrients 13:850. doi: 10. 3390/nu13030850

McGuire, S. O., Sortwell, C. E., Shukitt-Hale, B., Joseph, J. A., Hejna, M. J., and Collier, T. J. (2006). Dietary supplementation with blueberry extract improves survival of transplanted dopamine neurons. Nutr. Neurosci. 9, 251-258. doi: $10.1080 / 10284150601086134$

McNamara, R. K., Kalt, W., Shidler, M. D., McDonald, J., Summer, S. S., Stein, A. L., et al. (2018). Cognitive response to fish oil, blueberry, and combined supplementation in older adults with subjective cognitive impairment. Neurobiol. Aging 64, 147-156.

Middleton, L. E., Mitnitski, A., Fallah, N., Kirkland, S. A., and Rockwood, K. (2008). Changes in cognition and mortality in relation to exercise in late life: a population based study. PLoS One 3:e3124. doi: 10.1371/journal.pone.0003124

Miller, M. G., Hamilton, D. A., Joseph, J. A., and Shukitt-Hale, B. (2018). Dietary blueberry improves cognition among older adults in a randomized, doubleblind, placebo-controlled trial. Eur. J. Nutr. 57, 1169-1180. doi: 10.1007/ s00394-017-1400-8

Minghui, R. (2019). Risk Reduction of Cognitive Decline and Dementia. Geneva: World Health Organization. WHO guidelines.

Moco, S., Martin, F. P., and Rezzi, S. (2012). Metabolomics view on gut microbiome modulation by polyphenol-rich foods. J. Proteome Res. 11, 4781-4790. doi: $10.1021 / \mathrm{pr} 300581 \mathrm{~s}$

Morais, C., de Rosso, V., Estadella, D., and Pisani, L. (2016). Anthocyanins as inflammatory modulators and the role of the gut microbiota. J. Nutri. Biochem. $33,1-7$.

Mu, C., Yang, Y., and Zhu, W. (2016). Gut microbiota: the brain peacekeeper. Front. Microbiol. 7:345. doi: 10.3389/fmicb.2016.00345

Napoli, N., Shah, K., Waters, D. L., Sinacore, D. R., Qualls, C., and Villareal, D. T. (2014). Effect of weight loss, exercise, or both on cognition and quality of life in obese older adults. Am. J. Clin. Nutr. 100, 189-198. doi: 10.3945/ajcn.113. 082883

Nasreddine, Z. S., Phillips, N. A., Bedirian, V., Charbonneau, S., Whitehead, V., Collin, I., et al. (2005). The Montreal cognitive assessment, MoCA: a brief screening tool for mild cognitive impairment. J. Am. Geriatr. Soc. 53, 695-699.

Nieman, D., Gillitt, N., Chen, G., Zhang, Q., Sha, W., Kay, C. D., et al. (2020). Blueberry and/or banana consumption mitigate cytochrome P450 oxylipin generation during recovery from $75-\mathrm{km}$ cycling: a randomized trial. Front. Nutr. 7:121. doi: 10.3389/fnut.2020.00121

Nieman, D., and Pence, B. (2020). Exercise immunology: future directions. J. Sport Health Sci. 9, 432-445.

Nieman, D. C., Gillitt, N. D., Knab, A. M., Shanely, R. A., Pappan, K. L., Jin, F., et al. (2013). Influence of a polyphenol-enriched protein powder on exerciseinduced inflammation and oxidative stress in athletes: a randomized trial using a metabolomics approach. PLoS One 8:e72215. doi: 10.1371/journal.pone. 0072215

Nieman, D. C., Kay, C. D., Rathore, A. S., Grace, M. H., Strauch, R. C., Stephan, E. H., et al. (2018). Increased plasma levels of gut-derived phenolics linked to walking and running following two weeks of flavonoid supplementation. Nutrients 10:1718. doi: 10.3390/nu10111718

Nieman, D. C., Lila, M. A., and Gillitt, N. D. (2019). Immunometabolism: a multiomics approach to interpreting the influence of exercise and diet on the immune system. Annu. Rev. Food Sci. Technol. 10, 341-363. doi: 10.1146/annurev-food032818-121316

Nieman, D. C., Ramamoorthy, S., Kay, C. D., Goodman, C. L., Capps, C. R., Shue, Z. L., et al. (2017). Influence of ingesting a flavonoid-rich supplement on the 
metabolome and concentration of urine phenolics in overweight/obese women. J. Proteome Res. 16, 2924-2935.

Noble, E. E., Hsu, T. M., and Kanoski, S. E. (2017). Gut to brain dysbiosis: mechanisms linking Western diet consumption, the microbiome, and cognitive impairment. Front. Behav. Neurosci. 11:9. doi: 10.3389/fnbeh.2017.00009

Novotny, M., Klimova, B., and Valis, M. (2019). Microbiome and cognitive impairment: can any diets influence learning processes in a positive way? Front. Aging Neurosci. 11:170. doi: 10.3389/fnagi.2019.00170

Peet, R. (1974). The measurement of species diversity. Annu. Rev. Ecol. Syst. 5, 285-307.

Pereira-Caro, G., Oliver, C. M., Weerakkody, R., Singh, T., Conlon, M., Borges, G., et al. (2015). Chronic administration of a microencapsulated probiotic enhances the bioavailability of orange juice flavanones in humans. Free Radic. Biol. Med. $84,206-214$.

Piercy, K. L., and Troiano, R. P. (2018). Physical activity guidelines for Americans From the US department of health and human services. Circ. Cardiovasc. Qual. Outcomes 11:e005263.

Rajaram, S., Jones, J., and Lee, G. J. (2019). Plant-based dietary patterns, plant foods, and age-related cognitive decline. Adv. Nutr. 10(Suppl._4), S422-S436. doi: 10.1093/advances/nmz081

Rehfeld, K., Luders, A., Hokelmann, A., Lessmann, V., Kaufmann, J., Brigadski, T., et al. (2018). Dance training is superior to repetitive physical exercise in inducing brain plasticity in the elderly. PLoS One 13:e0196636. doi: 10.1371/ journal.pone. 0196636

Rooks, C. R., Thom, N. J., McCully, K. K., and Dishman, R. K. (2010). Effects of incremental exercise on cerebral oxygenation measured by near-infrared spectroscopy: a systematic review. Prog. Neurobiol. 92, 134-150. doi: 10.1016/ j.pneurobio.2010.06.002

Roopchand, D. E., Carmody, R. N., Kuhn, P., Moskal, K., Rojas-Silva, P., Turnbaugh, P. J., et al. (2015). Dietary polyphenols promote growth of the gut bacterium Akkermansia muciniphila and attenuate high-fat diet-induced metabolic syndrome. Diabetes 64, 2847-2858. doi: 10.2337/db14-1916

Rovio, S., Spulber, G., Nieminen, L. J., Niskanen, E., Winblad, B., Tuomilehto, J., et al. (2010). The effect of midlife physical activity on structural brain changes in the elderly. Neurobiol. Aging 31, 1927-1936. doi: 10.1016/j.neurobiolaging. 2008.10.007

Ruotolo, R., Minato, I., La Vitola, P., Artioli, L., Curti, C., Franceschi, V., et al. (2020). Flavonoid-derived human phenyl-gamma-valerolactone metabolites selectively detoxify amyloid-beta oligomers and prevent memory impairment in a mouse model of Alzheimer's disease. Mol. Nutr. Food Res. 2020:e1900890. doi: $10.1002 / \mathrm{mnfr} .201900890$

Saji, N., Murotani, K., Hisada, T., Tsuduki, T., Sugimoto, T., Kimura, A., et al. (2019). The relationship between the gut microbiome and mild cognitive impairment in patients without dementia: a cross-sectional study conducted in japan. Sci. Rep. 9:19227. doi: 10.1038/s41598-019-55851-y

Santoro, A., Brigidi, P., Gonos, E. S., Bohr, V. A., and Franceschi, C. (2014). Mediterranean diet and inflammaging in the elderly: the European project NU-AGE. preface. Mech. Ageing Dev. 13, 1-2. doi: 10.1016/j.mad.2014.01.006

Schell, J., Betts, N. M., Lyons, T. J., and Basu, A. (2019). Raspberries improve postprandial glucose and acute and chronic inflammation in adults with type 2 diabetes. Ann. Nutr. Metab. 74, 165-174. doi: 10.1159/000497226

Scheperjans, F., Aho, V., Pereira, P. A., Koskinen, K., Paulin, L., Pekkonen, E., et al. (2015). Gut microbiota are related to Parkinson's disease and clinical phenotype. Mov. Disord. 30, 350-358. doi: 10.1002/mds.26069

Schlegel, P., Novotny, M., Klimova, B., and Valis, M. (2019). "Muscle-gut-brain axis": can physical activity help patients with Alzheimer's disease due to microbiome modulation? J. Alzheimers Dis. 71, 861-878. doi: 10.3233/JAD190460

Shukitt-Hale, B., Bielinski, D. F., Lau, F. C., Willis, L. M., Carey, A. N., and Joseph, J. A. (2015). The beneficial effects of berries on cognition, motor behaviour and neuronal function in ageing. Br. J. Nutr. 114, 1542-1549. doi: 10.1017/ S0007114515003451

Small, B. J., Rawson, K. S., Martin, C., Eisel, S. L., Sanberg, C. D., McEvoy, C. L., et al. (2014). Nutraceutical intervention improves older adults' cognitive functioning. Rejuvenation Res. 17, 27-32. doi: 10.1089/rej.2013.1477

Socci, V., Tempesta, D., Desideri, G., De Gennaro, L., and Ferrara, M. (2017). Enhancing human cognition with cocoa flavonoids. Front. Nutr. 4:19. doi: $10.3389 /$ fnut.2017.00019
Sokolov, A. N., Pavlova, M. A., Klosterhalfen, S., and Enck, P. (2013). Chocolate and the brain: Neurobiological impact of cocoa flavanols on cognition and behavior. Neurosci. Biobehav. Rev. 37(10 Pt 2), 2445-2453. doi: 10.1016/j.neubiorev.2013. 06.013

Spencer, J. P., Vafeiadou, K., Williams, R. J., and Vauzour, D. (2012). Neuroinflammation: modulation by flavonoids and mechanisms of action. Mol. Aspects Med. 33, 83-97. doi: 10.1016/j.mam.2011.10.016

Spencer, J. P. E. (2009). Flavonoids and brain health: multiple effects underpinned by common mechanisms. Genes Nutr. 4, 243-250.

Strathearn, K. E., Yousef, G. G., Grace, M. H., Roy, S. L., Tambe, M. A., Ferruzzi, M. G., et al. (2014). Neuroprotective effects of anthocyanin- and proanthocyanidin-rich extracts in cellular models of Parkinsons disease. Brain Res. 1555, 60-77. doi: 10.1016/j.brainres.2014.01.047

Tomas-Barberan, F. A., Selma, M. V., and Espin, J. C. (2016). Interactions of gut microbiota with dietary polyphenols and consequences to human health. Curr. Opin. Clin. Nutr. Metab Care 19, 471-476. doi: 10.1097/MCO. 0000000000000314

Tooley, K. L. (2020). Effects of the human gut microbiota on cognitive performance, brain structure and function: a narrative review. Nutrients 12:3009. doi: $10.3390 /$ nu12103009

Tsubaki, A., Morishita, S., Hotta, K., Tokunaga, Y., Qin, W., Kojima, S., et al. (2021). Changes in the laterality of oxygenation in the prefrontal cortex and premotor area during a 20-min moderate-intensity cycling exercise. Adv. Exp. Med. Biol. 1269, 113-117. doi: 10.1007/978-3-030-48238-1_18

Tsukamoto, H., Suga, T., Ishibashi, A., Takenaka, S., Tanaka, D., Hirano, Y., et al. (2018). Flavanol-rich cocoa consumption enhances exercise-induced executive function improvements in humans. Nutrition 46, 90-96.

Tsukamoto, H., Takenaka, S., Suga, T., Tanaka, D., Takeuchi, T., Hamaoka, T., et al. (2017). Effect of exercise intensity and duration on post-exercise executive function. Med. Sci. Sports Exerc. 49, 774-784. doi: 10.1249/MSS. 0000000000001155

Tuohy, K. M., Conterno, L., Gasperotti, M., and Viola, R. (2012). Up-regulating the human intestinal microbiome using whole plant foods, polyphenols, and/or fiber. J. Agric. Food Chem. 60, 8776-8782. doi: 10.1021/jf205 3959

U.S. Department of Health and Human Services and U.S. Department of Agriculture (2015). 2015-2020 Dietary guidelines for Americans, 8th Edn. London: Pearson.

United States Census Bureau (2021). Population Projections. Suitland, MA: US Census Bureau.

Vauzour, D., Rendeiro, C., D’Amato, A., Waffo-Téguo, P., Richard, T., Mérillon, J. M., et al. (2021). Anthocyanins promote learning through modulation of synaptic plasticity related proteins in an animal model of ageing. Antioxidants 10:1235. doi: 10.3390/antiox 10081235

Voss, M. W., Nagamatsu, L. S., Liu-Ambrose, T., and Kramer, A. F. (2011). Exercise, brain, and cognition across the life span. J. Appl. Physiol. 111, 1505-1513. doi: 10.1152/japplphysiol.00210.2011

Wang, L., Gao, M., Kang, G., and Huang, H. (2021). The potential role of phytonutrients flavonoids influencing gut microbiota in the prophylaxis and treatment of inflammatory bowel disease. Front. Nutr. 8:798038. doi: 10.3389/ fnut.2021.798038

Wang, Y., and Dykes, G. A. (2021). Direct modulation of the gut microbiota as a therapeutic approach for Alzheimer's disease. CNS Neurol. Disord. Drug Targets 21, 14-25. doi: 10.2174/1871527320666210806165751

Warner, E. F., Smith, M. J., Zhang, Q., Raheem, K. S., O'Hagan, D., O'Connell, M. A., et al. (2017). Signatures of anthocyanin metabolites identified in humans inhibit biomarkers of vascular inflammation in human endothelial cells. Mol. Nutr. Food Res. 61:1700053. doi: 10.1002/mnfr.201700053

Weng, T., Pierce, G., Darling, W., and Voss, M. (2015). Differential effects of acute exercise on distinct aspects of executive function. Med. Sci. Sports Exerc. 47, 1460-1469. doi: 10.1249/MSS.0000000000000542

Westfall, S., Lomis, N., and Prakash, S. (2018). A novel polyphenolic prebiotic and probiotic formulation have synergistic effects on the gut microbiota influencing Drosophila melanogaster physiology. Artif. Cells Nanomed. Biotechnol. 46, 441455. doi: 10.1080/21691401.2018.1458731

Westfall, S., and Pasinetti, G. M. (2019). The gut microbiota links dietary polyphenols with management of psychiatric mood disorders. Front. Neurosci. 13:1196. doi: 10.3389/fnins.2019.01196 
Whyte, A. R., Cheng, N., Fromentin, E., and Williams, C. (2018). A randomized, double-blinded, placebo-controlled study to compare the safety and efficacy of low dose enhanced wild blueberry powder and wild blueberry extract (ThinkBlue ${ }^{\mathrm{TM}}$ ) in maintenance of episodic and working memory in older adults. Nutrients 10:660.

Whyte, A. R., Rahman, S., Bell, L., Edirisinghe, I., Krikorian, R., Williams, C. M., et al. (2020). Improved metabolic function and cognitive performance in middle-aged adults following a single dose of wild blueberry. Eur. J. Nutr. 60, 1521-1536. doi: 10.1007/s00394-020-02336-8

Williams, C. M., El Mohsen, M. A., Vauzour, D., Rendeiro, C., Butler, L. T., Ellis, J. A., et al. (2008). Blueberry-induced changes in spatial working memory correlate with changes in hippocampal CREB phosphorylation and brainderived neurotrophic factor (BDNF) levels. Free Radic. Biol. Med. 45, 295-305. doi: 10.1016/j.freeradbiomed.2008.04.008

Williams, R. J., and Spencer, J. P. (2012). Flavonoids, cognition, and dementia: actions, mechanisms, and potential therapeutic utility for Alzheimer disease. Free Radic. Biol. Med. 52, 35-45. doi: 10.1016/j.freeradbiomed.2011. 09.010

Williamson, G., Kay, C., and Crozier, A. (2018). The bioavailability, transport, and bioactivity of dietary flavonoids: a review from a historical perspective. Compr. Rev. Food Sci. Food Saf. 17, 1054-1112.

Zhou, X. L., Wang, L. N., Wang, J., Zhou, L., and Shen, X. H. (2020). Effects of exercise interventions for specific cognitive domains in old adults with mild cognitive impairment: a meta-analysis and subgroup analysis of randomized controlled trials. Medicine 99:e20105. doi: 10.1097/MD.00000000000 20105
Zhu, Q., Jiang, S., and Du, G. (2020). Effects of exercise frequency on the gut microbiota in elderly individuals. Microbiologyopen 9:e1053. doi: 10.1002/ mbo3.1053

Zhu, W., Wadley, V., Howard, V., Hutto, B., Blair, S., and Hooker, S. (2016). Objectively measured physical activity and cognitive function in older adults. Med. Scie. Sports Exer. 49, 47-53. doi: 10.1249/MSS.0000000000001079

Zmora, N., Suez, J., and Elinav, E. (2019). You are what you eat: diet, health and the gut microbiota. Nat. Rev. Gastroenterol. Hepatol. 16, 35-56. doi: 10.1038/ s41575-018-0061-2

Conflict of Interest: The authors declare that the research was conducted in the absence of any commercial or financial relationships that could be construed as a potential conflict of interest.

Publisher's Note: All claims expressed in this article are solely those of the authors and do not necessarily represent those of their affiliated organizations, or those of the publisher, the editors and the reviewers. Any product that may be evaluated in this article, or claim that may be made by its manufacturer, is not guaranteed or endorsed by the publisher.

Copyright (C) 2022 Cheatham, Nieman, Neilson and Lila. This is an open-access article distributed under the terms of the Creative Commons Attribution License (CC BY). The use, distribution or reproduction in other forums is permitted, provided the original author(s) and the copyright owner(s) are credited and that the original publication in this journal is cited, in accordance with accepted academic practice. No use, distribution or reproduction is permitted which does not comply with these terms. 